\title{
Femtosecond laser ablation of enamel
}

\author{
Quang-Tri Le \\ Caroline Bertrand \\ Rui Vilar
}




\title{
Femtosecond laser ablation of enamel
}

\author{
Quang-Tri Le, ${ }^{a, b, *}$ Caroline Bertrand, ${ }^{b}$ and Rui Vilar ${ }^{a}$ \\ aLisbon University, Instituto Superior Técnico and CeFEMA Center of Physics and Engineering of Advanced Materials, Avenida Rovisco Pais, \\ 1049-001 Lisboa, Portugal \\ bLaboratoire ICMCB-CNRS-UPR9048, 87, Avenue du Dr Albert Schweitzer, 33608 PESSAC Cedex, France
}

\begin{abstract}
The surface topographical, compositional, and structural modifications induced in human enamel by femtosecond laser ablation is studied. The laser treatments were performed using a Yb:KYW chirped-pulseregenerative amplification laser system (560 fs and $1030 \mathrm{~nm}$ ) and fluences up to $14 \mathrm{~J} / \mathrm{cm}^{2}$. The ablation surfaces were studied by scanning electron microscopy, grazing incidence x-ray diffraction, and micro-Raman spectroscopy. Regardless of the fluence, the ablation surfaces were covered by a layer of resolidified material, indicating that ablation is accompanied by melting of hydroxyapatite. This layer presented pores and exploded gas bubbles, created by the release of gaseous decomposition products of hydroxyapatite $\left(\mathrm{CO}_{2}\right.$ and $\left.\mathrm{H}_{2} \mathrm{O}\right)$ within the liquid phase. In the specimen treated with $1-\mathrm{kHz}$ repetition frequency and $14 \mathrm{~J} / \mathrm{cm}^{2}$, thickness of the resolidified material is in the range of 300 to $900 \mathrm{~nm}$. The micro-Raman analysis revealed that the resolidified material contains amorphous calcium phosphate, while grazing incidence $\mathrm{x}$-ray diffraction analysis allowed detecting traces of a calcium phosphate other than hydroxyapatite, probably $\beta$-tricalcium phosphate $\mathrm{Ca}_{3}\left(\mathrm{PO}_{4}\right)_{2}$, at the surface of this specimen. The present results show that the ablation of enamel involves melting of enamel's hydroxyapatite, but the thickness of the altered layer is very small and thermal damage of the remaining material is negligible. $\odot 2016$ Society of Photo-Optical Instrumentation Engineers (SPIE) [DOI: 10.1117/1.JBO.21.6.065005]
\end{abstract}

Keywords: femtosecond laser; enamel; surface modification; ablation.

Paper 160126R received Mar. 1, 2016; accepted for publication Jun. 1, 2016; published online Jun. 22, 2016.

\section{Introduction}

Since their introduction to commercial use in the 1960 s, lasers have been investigated as an alternative to conventional mechanical methods in dental caries treatment. The initial research efforts carried out using continuous wave or pulsed $\mathrm{CO}_{2}$ lasers (wavelength ranging from 9.3 to $\left.10.6 \mu \mathrm{m}\right)^{1-4}$ and $\mathrm{Nd}$ :YAG lasers with wavelength $1064 \mathrm{~nm}^{5,6}$ failed to satisfy the medical requirements. Continuous wave lasers do not allow controlling precisely the laser-material interaction time, often leading to extensive melting, carbonization, and thermomechanical cracking of the teeth and, in extreme cases, necrosis of the pulpal tissue. ${ }^{7}$ Pulsed $\mathrm{CO}_{2}$ and Nd:YAG lasers, with pulse duration in the $10^{-3}$ to $10^{-9} \mathrm{~s}$ range, allow reducing thermal damage but, due to the relatively long interaction time, heat transfer from the interaction region to the tooth bulk is still important, leading to melting, thermomechanical cracking, ${ }^{1,3,8}$ loss of collagen, and decomposition of hydroxyapatite. ${ }^{9}$ Moreover, the ablation rates achieved with those lasers are insufficient for the clinical applications. ${ }^{10}$

Several approaches have been tested to improve these results. Er,Cr:YSGG and Er:YAG lasers, which emit at 2.79 and $2.94 \mu \mathrm{m}$, respectively, allow achieving relatively high ablation rates with minimal thermal degradation of the teeth and became the main type of lasers used in clinics. ${ }^{11,12}$ This is due to the fact that their radiation wavelengths are within a strong absorption band of water. ${ }^{13,14}$ As a result, radiation is efficiently absorbed by the water contained in the tissues, which warms up and boils explosively, leading to the mechanical disruption of the tissue matrix. ${ }^{15-17}$ This ablation mechanism minimizes heat transferred

*Address all correspondence to: Quang-Tri Le, E-mail: quang.le@tecnico. ulisboa.pt to the tooth, but it has been reported that the shockwave created by the rapid water evaporation may lead to the formation of subsuperficial cracks. ${ }^{18}$ These thermomechanical effects can be minimized by a proper selection of the processing parameters and by using a cooling water spray. ${ }^{19}$ Another approach to reduce thermal effects is using UV lasers. ${ }^{20-22}$ Eugenio et al. ${ }^{22}$ and Sivakumar et al. ${ }^{23}$ showed that intertubular dentin can be ablated by a $\mathrm{KrF}$ excimer laser with a $248-\mathrm{nm}$ radiation wavelength and 30-ns pulse duration with negligible thermal effects and no degradation of the remaining material. The ablation mechanism is predominantly photochemical for intertubular dentin and photothermal for peritubular dentin. ${ }^{22,24}$ However, excimer lasers are not suitable for clinical application due to their low ablation rate and the potential health hazard caused by exposure to intense UV radiation. A more effective method to reduce thermal effects is using ultrafast lasers. Femtosecond lasers have been shown to ablate dentin ${ }^{25-27}$ and bone ${ }^{28}$ efficiently and with negligible thermal degradation of the underlying tissue. For such short pulse durations, the interaction time is shorter than the characteristic energy relaxation times such as the electron-lattice energy transfer time and heat conduction time. ${ }^{28}$ Therefore, no energy is transferred to the lattice during the laser pulse and most of the absorbed radiation energy is spent on the ablation process and carried away by the ablated material. Moreover, since femtosecond laser radiation absorption by dielectric materials is highly nonlinear, the interaction zone is confined to a very small region, similar to the focal volume. With an ultrafast laser, the energy efficiency is very high and the ablation rates achieved are higher than those obtained with nanosecond pulse duration infrared ${ }^{29}$ and UV lasers. ${ }^{30}$ Surface analysis of the ultrafast laser ablation surfaces

$1083-3668 / 2016 / \$ 25.00$ (c) 2016 SPIE 
of dentin ${ }^{26,27}$ and bone ${ }^{28}$ revealed that no significant changes in the tissue composition and structure occurred.

The ablation of enamel by femtosecond lasers was studied by a number of authors ${ }^{25,27,29,31}$ but no detailed characterization on the ablated surfaces was performed. In the present paper, the chemical and structural modifications induced by ultrafast laser ablation on enamel are studied and related with enamel ablation mechanisms.

\section{Materials and Methods}

\subsection{Sample Preparation}

The protocol for the collection of enamel specimens used in the present study was approved by the local ethics committee (process DC 2014/04). Enamel specimens were cut from recently extracted human molars by using a low speed diamond saw (Accutom-50 ${ }^{\circledR}$, Struers (C)). One of their faces was polished under flowing water using a 600 to 2500 grit sequence of $\mathrm{SiC}$ papers. The samples were prepared the day before the laser experiments and stored in distilled water at $4^{\circ} \mathrm{C}$. Prior to the laser treatment, they were extracted from the liquid and gently wiped with a clean tissue paper to remove the water excess.

The laser experiments were performed in ambient atmosphere, using a Yb:KYW chirped-pulse-regenerative amplification laser system with 560-fs pulse duration and 1030-nm

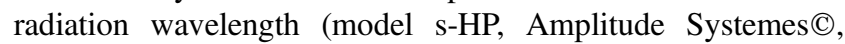
France). The Gaussian laser beam was perpendicular to the specimen and focused on its surface with a $100-\mathrm{mm}$ focal length lens, giving a spot radius of $35 \mu \mathrm{m}$. By varying the average laser beam power, radiation fluences in the range of 3 to $14 \mathrm{~J} / \mathrm{cm}^{2}$ were obtained. The pulse energy was determined from the average laser beam power, measured with an Ophir Photonics model 10ASHV1.1 RoHS power meter (Jerusalem, Israel).

The enamel ablation threshold was determined by the $D^{2}$ and the D-scan methods proposed by $\mathrm{Liu}^{32}$ and Machado et al., ${ }^{33}$ respectively. For the $D^{2}$ method, craters were created with a single laser pulse and a wide range of pulse energies. The diameter, $D$, of these craters was measured on calibrated scanning electron microscopy images. According to Liu, ${ }^{32}$ the relation between the diameter of the craters and the fluence, $F$, is given as

$D^{2}=2 w_{0}^{2} \ln \left(\frac{F}{F_{\mathrm{th}}}\right)$,

where $F_{\text {th }}$ is the single laser pulse ablation threshold. Equation (1) implies that $D^{2}$ varies linearly with $\ln (F)$, so the value of the ablation threshold can be estimated by extrapolating a plot of $D^{2}$ versus $\ln (F)$ to zero.

The D-scan method requires creating linear laser tracks by moving the sample simultaneously in the $X X$ and $Z Z$ directions at constant scanning speeds across the laser beam's focus. ${ }^{33}$ The width of these tracks reaches a minimum when the sample surface is at the focus and increases with increasing distance between this surface and the focal plane, reaching a maximum when the fluence is equal to the ablation threshold. The relationship between the material ablation threshold $F_{\text {th }}$, the pulse energy $E_{\mathrm{o}}$, and the maximum width of the ablated track $\rho_{\max }$ is given as ${ }^{33}$
$F_{\text {th }}=0.117 \cdot \frac{E_{0}}{\rho_{\max }^{2}}$.

The ablation threshold value determined by this method corresponds to multiple pulse conditions because pulse overlapping cannot be avoided. The number of laser pulses incident on each point of the track, $N$, can be calculated as ${ }^{33}$

$N=1.8 \cdot \frac{f \cdot \rho_{\max }}{v_{x}}$,

where $f$ is the pulse repetition rate and $v_{x}$ is the sample velocity in the scanning direction.

The laser tracks were inscribed with constant pulse energy and frequency (150 $\mu \mathrm{J}$ and $1 \mathrm{kHz}$, respectively) and scanning speeds ranging from 0.1 to $2 \mathrm{~mm} / \mathrm{s}$. The specimen was moved by a computer-controlled $X Y$ stage (PI miCos ${ }^{\circledR}$, Eschbach $($, Germany). The maximum width of the ablation tracks was measured on optical microscopy images. The results reported are the average of three experiments.

The morphology and structure of enamel ablation surfaces were studied on specimens treated with a pulse repetition rate of $1 \mathrm{kHz}$ and a scanning velocity of $5 \mathrm{~mm} / \mathrm{s}$. The surface treatment was performed by moving the specimen in the $X X$ direction to create a linear track. After completing the track, the specimen was returned to the origin of the movement, moved $0.01 \mathrm{~mm}$ in the $Y Y$ direction and the linear scan in the $X X$ direction repeated. These steps were repeated until complete surface coverage was achieved. Experiments were performed with fluences of 4,9 , and $14 \mathrm{~J} / \mathrm{cm}^{2}$. The average number of pulses per point of the surface was 403 .

\subsection{Sample Characterization}

The surface morphology was investigated in the secondary electron imaging mode using a JEOL $7001^{\circledR}$ (JEOL $\odot$, Tokyo, Japan) field-emission gun scanning electron microscope (SEM) operated at an acceleration voltage of $10 \mathrm{kV}$. To avoid charging effects, the samples were coated with a highly conductive $\mathrm{Pd} / \mathrm{Au}$ film prior to the observation. For cross-sectional SEM examination of the laser-treated specimens, the specimens were immersed in liquid $\mathrm{N}_{2}$ and, then, broken with a hammer. The surface chemical constitution of enamel surfaces before and after the laser treatment was studied by micro-Raman spectroscopy and x-ray diffraction. Micro-Raman analysis was performed with an XploRA ${ }^{\circledR}$ Confocal Raman system (Horiba Scientific $\left({ }^{\circ}\right.$, Japan). The Raman radiation was excited by a 5-mW, 786-nm radiation wavelength laser beam focused at the specimen's surface by a $50 \times$ microscope objective, which allows a surface region with thickness of $7 \mu \mathrm{m}$ to be analyzed. The radiation emitted by the specimens was analyzed by a spectrometer with a $1200 \mathrm{~g} / \mathrm{mm}$ grating and spectral resolution better than $1 \mathrm{~cm}^{-1}$. Before spectra acquisition, the Raman spectrometer was calibrated using a Si sample. X-ray diffraction was performed with a Bruker D8 Advance diffractometer equipped with a Goebel mirror, using $0.15418 \mathrm{~nm}$ wavelength $\mathrm{Cu}$ radiation. The angle of incidence was varied in the range of 0.2 deg to $1 \mathrm{deg}$ in order to analyze different depths of material. The $2 \theta$ angle was varied in the range of $10 \mathrm{deg}$ to $80 \mathrm{deg}$ with a step of $0.02 \mathrm{deg}$ and acquisition time of $10 \mathrm{~s} / \mathrm{step}$. The laser-treated surfaces were analyzed in the original condition and after ultrasonication to eliminate loosely attached ablation debris. 


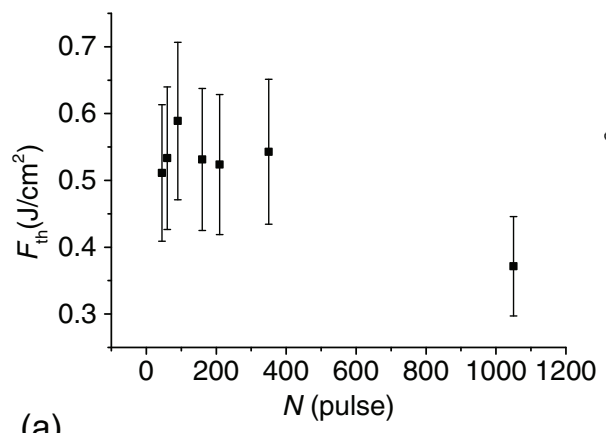

(a)

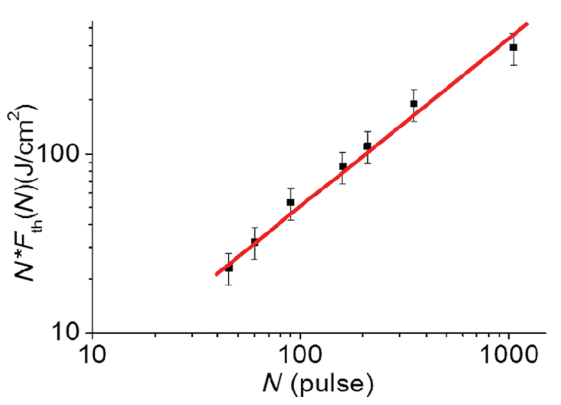

(b)

Fig. 1 (a) Variation of the ablation threshold with number of pulses and (b) plot of the accumulated fluence versus number of pulses.

\section{Results}

\subsection{Ablation Threshold}

The ablation threshold, calculated using the D-scan method described in Sec. 2, decreases with increasing number of laser pulses [Fig. 1(a)], indicating the existence of an incubation effect. $^{28}$ This effect was explained by the formation of defects in the material for radiation intensities below the ablation threshold, which create electronic energy states within the energy gap that facilitate radiation absorption for subsequent laser pulses. ${ }^{34}$ The single pulse ablation threshold and the incubation coefficient were calculated from multiple pulse experiments' results using the equations proposed by Jee et al. ${ }^{34}$

$F_{\text {th }}(N)=F_{\text {th }}(1) \cdot N^{S-1}$,

$N \cdot F_{\mathrm{th}}(N)=F_{\mathrm{th}}(1) \cdot N^{S}$,

where $F_{\text {th }}(N)$ and $F_{\text {th }}(1)$ are the ablation threshold values for $N$ pulse and a single pulse, respectively, and $S$ is the incubation factor, which characterizes incubation.

The relationship between the accumulated fluence $N \cdot F_{\text {th }}(N)$ and number of pulses $N$ is depicted in Fig. 1(b). The slope of the line represents the incubation factor $S$. The calculated values of the single pulse ablation threshold and of the incubation coefficient are $3.2 \pm 0.3 \mathrm{~J} / \mathrm{cm}^{2}$ and $0.68 \pm 0.08$, respectively.

A plot of $D^{2}$ versus $\operatorname{Ln}(F)$ for single pulse ablation experiments ( $F$ and $D$ are fluence and diameter of the craters,

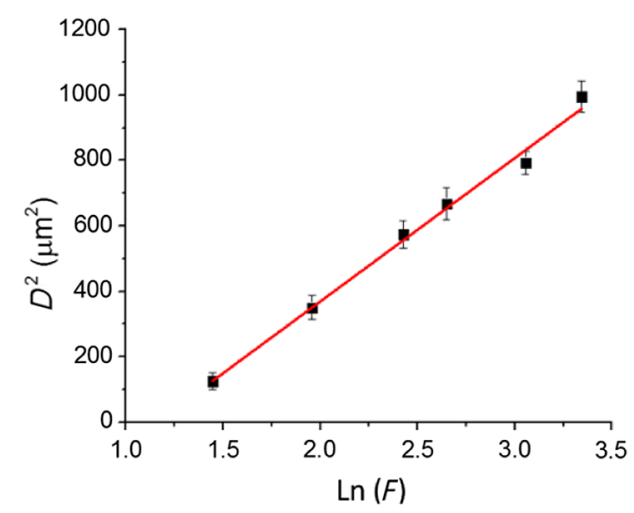

Fig. 2 Semilogarithmic plot of $D^{2}$ as a function of fluence and linear fitting of the experimental data. respectively) is shown in Fig. 2. The single pulse ablation threshold, determined by extrapolating $D^{2}$ to zero, is $3.4 \pm$ $0.2 \mathrm{~J} / \mathrm{cm}^{2}$, in good agreement with the value determined by the D-scan method.

\subsection{Morphology of the Laser-Treated Specimens}

The morphology of enamel surfaces treated with five laser pulses and fluences of 4,9 , and $14 \mathrm{~J} / \mathrm{cm}^{2}$ are shown in Figs. 3(a)-3(f). The surface is covered by a resolidified material layer, which presents round pores and traces of collapsed gas bubbles, showing that the tissue was melted and vapor was generated within the liquid, leading to the formation of gas bubbles that collapsed upon reaching the surface. The continuity and thickness of the resolidified layer and the size of collapsed gas bubbles increase with increasing fluence, as shown in Figs. 3(c)-3(f), indicating that surface temperature increases with laser fluence.

The surface morphology changes drastically when the laser beam is scanned continuously [Figs. 4(a), 4(c), and 4(e)]. The laser-treated surfaces are covered with ablation debris, mostly aggregated in clusters, and resolidified droplets. The thickness of this layer and the size of the clusters increase with the fluence, and particles aggregate with $\sim 3$ to $4 \mu \mathrm{m}$ in diameter are observed for $14 \mathrm{~J} / \mathrm{cm}^{2}$. The layer of ablation debris layer of the specimens treated with 4 and $9 \mathrm{~J} / \mathrm{cm}^{2}$ is poorly adhered to the surface and easily removed by ultrasonication, exposing a layer of molten material underneath [Figs. 4(b) and 4(d)]. The layer formed at $14 \mathrm{~J} / \mathrm{cm}^{2}$ adheres better to the surface and a significant amount of ablation debris remains after ultrasonication [Fig. 4(f)]. The observation of the cross-section of a specimen treated with $14 \mathrm{~J} / \mathrm{cm}^{2}$ (Fig. 5) reveals a layer of resolidified material, with a thickness varying in the range of 300 to $900 \mathrm{~nm}$.

\subsection{Surface Constitution}

\subsubsection{XRD results}

The x-ray diffractograms of polished and laser-treated enamel surfaces are shown in Fig. 6. The peaks of untreated enamel can be indexed as nonstoichiometric hydroxyapatite, according to JCPDS card number 09-0432. ${ }^{28}$ The diffractograms of the specimens treated with 4 and $9 \mathrm{~J} / \mathrm{cm}^{2}$ are similar to the diffractogram of the polished specimen, but new peaks appear at $36.2 \mathrm{deg}$ and $42.4 \mathrm{deg}$ in the specimen treated with $14 \mathrm{~J} / \mathrm{cm}^{2}$, which indicate that new phases formed as a result of the laser treatment. These peaks could be indexed as the (131) 

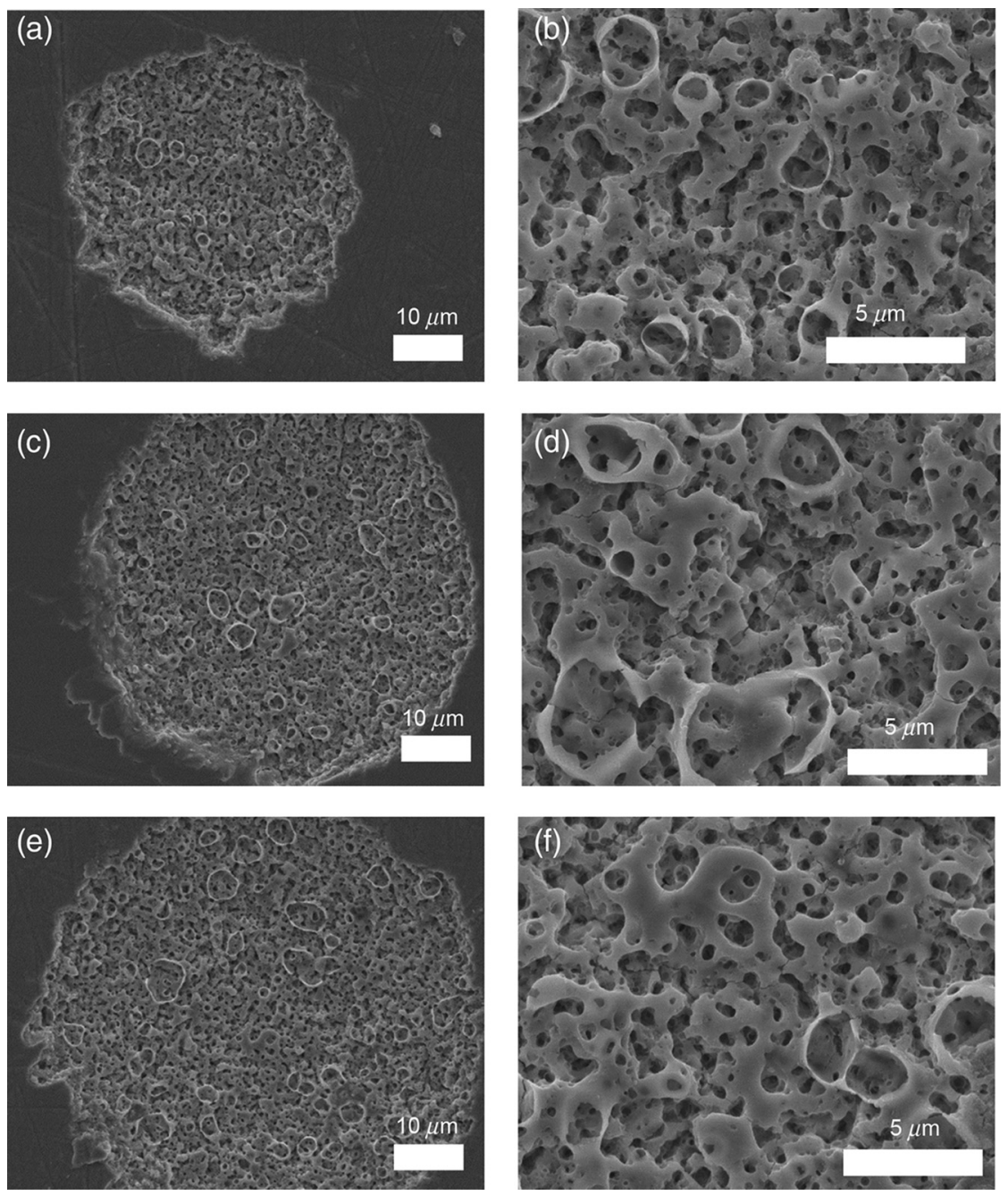

Fig. 3 Morphology of craters created on enamel surface using five laser pulses with radiation fluences of (a, b) $4 \mathrm{~J} / \mathrm{cm}^{2}$, (c, d) $9 \mathrm{~J} / \mathrm{cm}^{2}$, and $(e, f) 14 \mathrm{~J} / \mathrm{cm}^{2}$.

and (303) diffraction peaks of $\beta$-tricalcium phosphate $\mathrm{Ca}_{3}\left(\mathrm{PO}_{4}\right)_{2}$ (TCP, JCPDS card number 09-0169), ${ }^{35}$ respectively, but the diffraction pattern of this compound presents two intense peaks at $\sim 31.5 \mathrm{deg}$ and $34.5 \mathrm{deg}$, which are not seen in Fig. 6. On the other hand, these peaks cannot be attributed to the other two compounds that may form by the decomposition of hydroxyapatite at high temperature, tetracalcium phosphate $\mathrm{Ca}_{4}\left(\mathrm{PO}_{4}\right)_{2} \mathrm{O}$ (TTCP, JCDPS 70-1379), and calcium pyrophosphate $\beta-\mathrm{Ca}_{2} \mathrm{P}_{2} \mathrm{O}_{7}$ (JCDPS 9-0346). ${ }^{36}$ Therefore, we tentatively ascribe them to the (131) and (303) diffraction peaks of $\beta$-TCP. ${ }^{35}$ In order to investigate the depth distribution of this phase, GIXRD analysis was performed with several angles of incidence between $0.2 \mathrm{deg}$ and $1 \mathrm{deg}$ with $0.2 \mathrm{deg}$ step. The diffractograms normalized to the peak at $25.8 \mathrm{deg}$, corresponding to the diffraction of enamel apatite are shown in Fig. 7(a). The regions around the newly formed peaks are magnified and displayed in Fig. 7(b). The intensities of the peaks at $36 \mathrm{deg}$ and $42 \mathrm{deg}$ decrease for increasing incidence angle, showing that $\beta$-TCP exist mainly near the surface, probably in the ablation debris and in the resolidified layer.

\subsubsection{Raman results}

The Raman spectra of the enamel specimens, normalized to the strongest peak, at $960 \mathrm{~cm}^{-1}$, which corresponds to the $\nu_{1}$ vibration of the phosphate group in hydroxyapatite, ${ }^{28}$ are shown in Fig. 8. The peaks at 470, 530, and 1025 to $1085 \mathrm{~cm}^{-1}$ can be assigned to $\nu_{2}, \nu_{4}$, and $\nu_{3}$ vibrational modes of the phosphate group. ${ }^{37}$ The peak at $1070 \mathrm{~cm}^{-1}$, which corresponds to the $\nu_{1}$ vibration of the carbonate group, ${ }^{28,38}$ is also observed due to the partial replacement of hydroxyl and phosphate anions by the carbonate anion.

The relative intensity of the $\nu_{1}$ peak of $\mathrm{PO}_{4}^{3-}$ decreases with increasing fluence, which indicates the formation of nanocrystalline and/or amorphous calcium phosphate (ACP) at the surface of the sample. ${ }^{39,40}$ The presence of ACP is supported by the increase of relative intensity in the region around $950 \mathrm{~cm}^{-1}$ in the spectra of the laser-treated specimens, which corresponds to the $\nu_{1}$ Raman peak of that compound. ${ }^{39-41}$ This peak is still present in the Raman spectrum of the specimen treated with $14 \mathrm{~J} / \mathrm{cm}^{2}$ after ultrasonication, but its relative intensity decreases (Fig. 9), showing that ACP exists in the ablation debris. Raman spectra of the specimens treated with 4 and $9 \mathrm{~J} / \mathrm{cm}^{2}$ also show traces of ACP even after all the ablation debris were removed. The results show that the resolidified material also contains ACP.

\section{Discussion}

The enamel single pulse ablation threshold of enamel determined in the present work by $\mathrm{D}-\mathrm{scan}^{33}$ and $D^{232}$ methods 

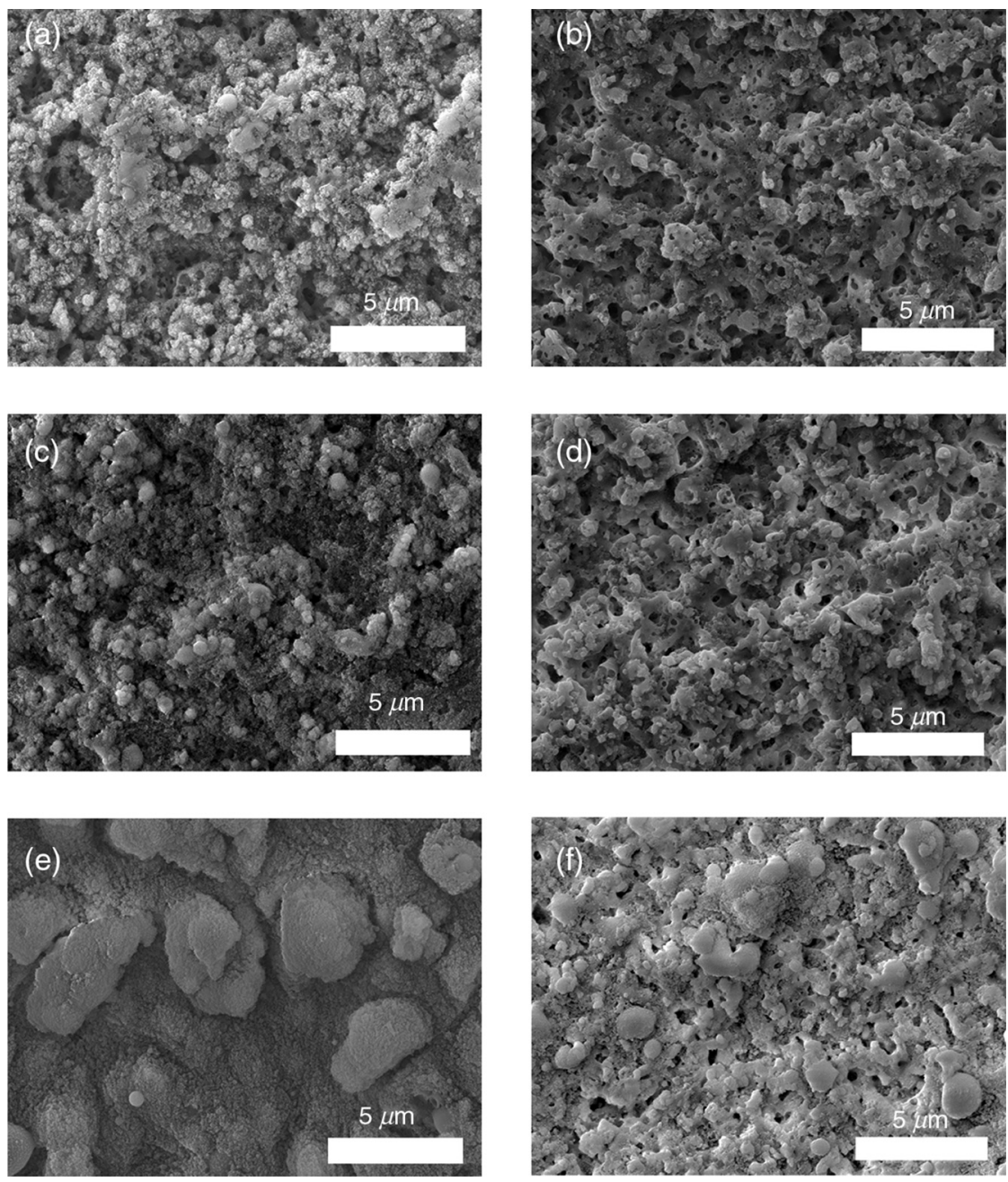

Fig. 4 Morphology of laser-treated enamel surfaces before and after ultrasonication. Fluences: (a, b) $4 \mathrm{~J} / \mathrm{cm}^{2}$, (c, d) $9 \mathrm{~J} / \mathrm{cm}^{2}$, and (e, f) $14 \mathrm{~J} / \mathrm{cm}^{2}$.

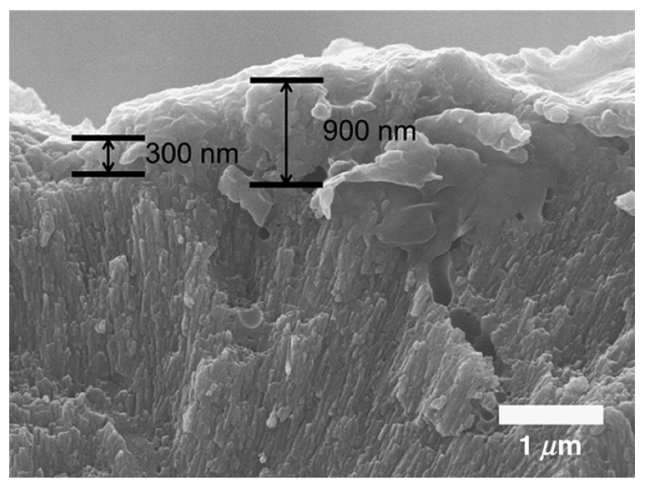

Fig. 5 Cross-section of a specimen treated with $14 \mathrm{~J} / \mathrm{cm}^{2}$.

were $3.2 \pm 0.3$ and $3.4 \pm 2 \mathrm{~J} / \mathrm{cm}^{2}$, respectively. This value cannot be directly compared with those previously reported in the literature because they were determined for multiple pulse ablation. $^{25,42}$ Krüger et al. ${ }^{42}$ found a threshold of $0.6 \mathrm{~J} / \mathrm{cm}^{2}$ for 100 pulses of $615 \mathrm{~nm}$ radiation wavelength with $350 \mathrm{fs}$ duration, while Neev et al. ${ }^{25}$ reported a value of $0.7 \mathrm{~J} / \mathrm{cm}^{2}$ for 100 pulses, 350-fs pulse duration, and 1050-nm radiation wavelength. By substituting the data reported by Neev et al. ${ }^{25}$ and Krüger et al. ${ }^{42}$ in Eq. (5) and using the incubation coefficient value found in the present work $(0.68 \pm 0.08)$, single pulse ablation thresholds of 3.0 and $2.7 \mathrm{~J} / \mathrm{cm}^{2}$, respectively, were obtained, in good agreement with our result. On the contrary, the value obtained here is much higher than the single pulse ablation threshold proposed by $\mathrm{Ji}$ et al. ${ }^{31}\left(0.58 \mathrm{~J} / \mathrm{cm}^{2}\right)$ for 800 -nm wavelength, and 85 -fs pulse duration. ${ }^{31}$ This difference could be explained by the much shorter pulse duration and reduced wavelength of the laser used by Ji et al., ${ }^{31}$ but Rode et al. ${ }^{29}$ found a single pulse ablation threshold of $2.2 \mathrm{~J} / \mathrm{cm}^{2}$ using laser parameters similar to Ji et al. (805-nm laser wavelength and 92-fs pulse duration). The large scatter of the ablation threshold values found in the literature reflects sample to sample variations typical for dental tissues and other natural materials. The calculated value of the incubation coefficient $0.68 \pm 0.08$ is in good agreement with the value reported by Cangueiro et al. ${ }^{28}$ for bone.

Several mechanisms have been proposed for the ultrashort laser ablation of dielectric materials. At low radiation intensities, Coulomb explosion is predominant, ${ }^{43,44}$ which is characterized by the laser-induced emission of photoelectrons from the surface of dielectric materials at the beginning of the laser pulse, resulting in the buildup of a high positive charge density. ${ }^{43}$ Once the electrostatic repulsion forces between the positive ions exceed the binding forces, these ions are ejected. ${ }^{43}$ In this ablation mechanism, material is removed mainly by the electrostatic 


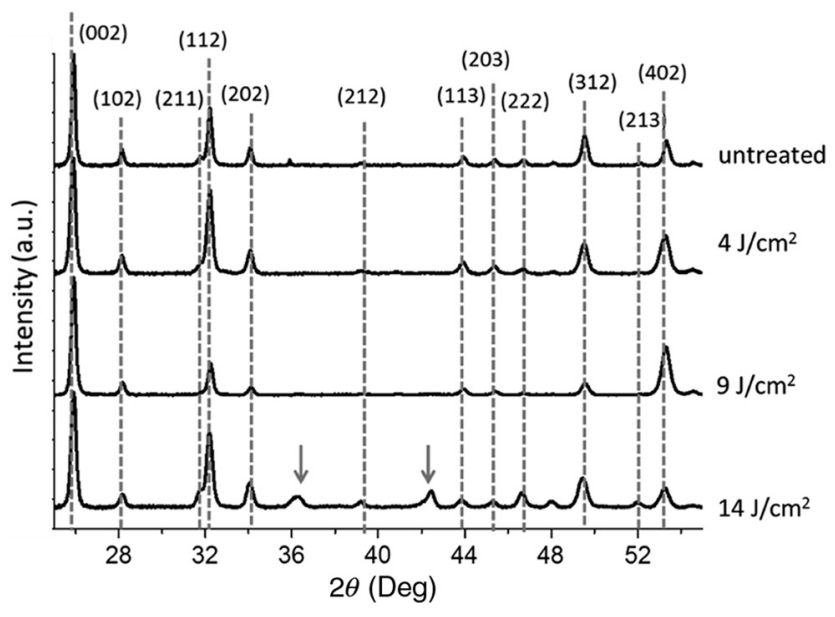

Fig. 6 Diffractograms of polished and laser-treated enamel. Angle of incidence: $0.2 \mathrm{deg}$. The indexed peaks correspond to hydroxyapatite. The arrows indicate the peaks corresponding to $\beta$-TCP, present in the specimen treated with $14 \mathrm{~J} / \mathrm{cm}^{2}$.

process and thermal effects are neligible. ${ }^{44}$ At higher radiation intensities, part of the absorbed energy is transferred to the lattice by electron-phonon interactions, leading to extremely fast heating of the material, which transforms into a highly overheated liquid. ${ }^{44,45}$ Eventually, the liquid phase may undergo different ablation processes including liquid spallation and phase explosion. ${ }^{44,46}$ In the present study, a thin layer of resolidified material is observed at the surface of the laser-treated specimens, independently of the processing parameters used. Melting of enamel indicates that a surface temperature above $1600^{\circ} \mathrm{C}$ was reached, $, 9,47$ and that the ablation process was dominated by thermal processes. On the other hand, the pores and traces of burst bubbles observed in the resolidified layer (Fig. 3) show that the liquid decomposed, resulting in the release of gas. According to Holcomb and Young, ${ }^{48}$ enamel releases gaseous water and $\mathrm{CO}_{2}$ in the solid state at temperatures of $100^{\circ} \mathrm{C}$ and $250^{\circ} \mathrm{C}$, respectively, which are much lower than the melting point of the tissue. ${ }^{9,48}$ These reactions cannot occur in the present case due to the extremely fast heating rate and decomposition of enamel only occurs after melting, leading to gas release within the liquid.

The ablation behavior of enamel observed in this study is essentially different from the ablation behavior of bone ${ }^{28}$ and

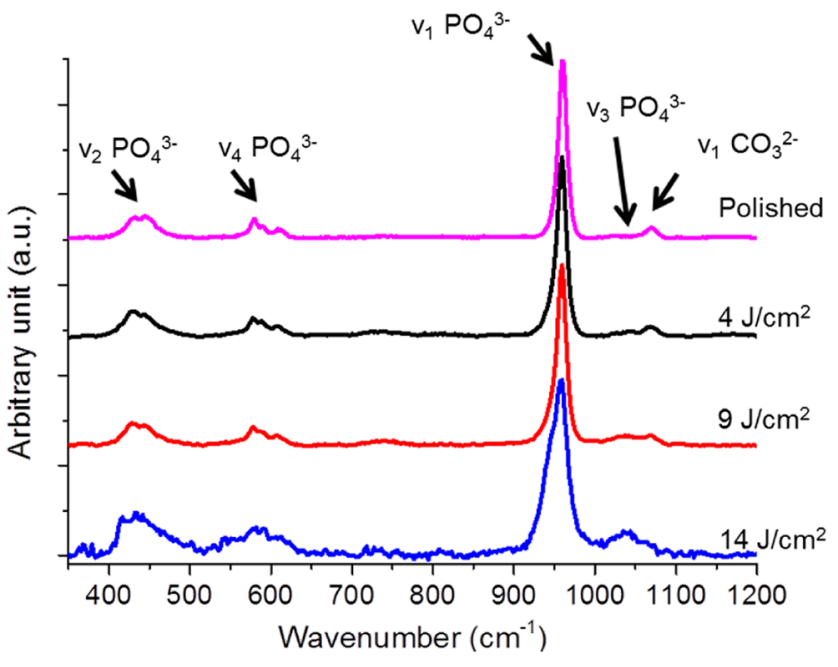

Fig. 8 Raman spectra of polished and laser-treated enamel.

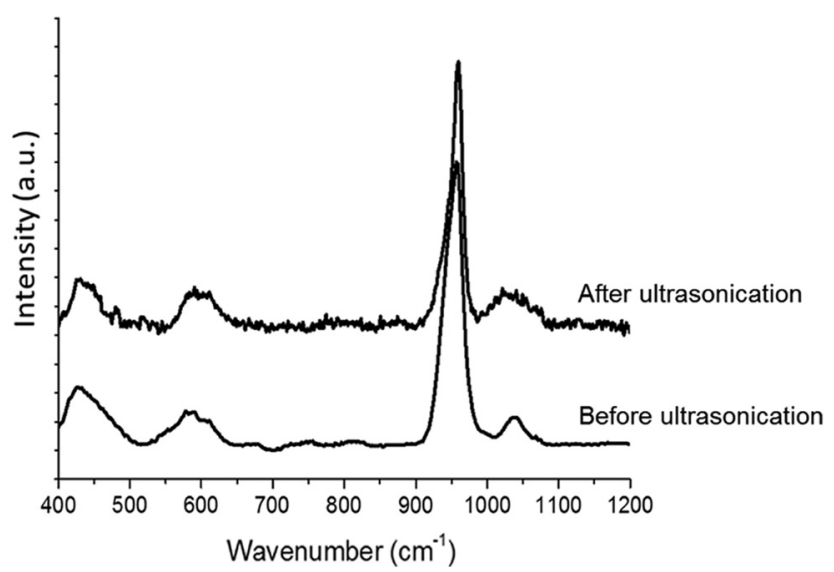

Fig. 9 Raman spectra of specimen treated with $14 \mathrm{~J} / \mathrm{cm}^{2}$ before and after ultrasonication.

dentin ${ }^{26}$ with the same laser system and fluences in the ranges of 0.55 to $2.18 \mathrm{~J} / \mathrm{cm}^{2}$ and 1 to $3 \mathrm{~J} / \mathrm{cm}^{2}$, respectively. The values of the single pulse ablation threshold of dentin and bone are significantly lower than enamel's $\left(0.6,0.79\right.$, and $3.2 \mathrm{~J} / \mathrm{cm}^{2}$, respectively). On the other hand, no melting was observed in both cases. This difference in ablation behavior can be explained

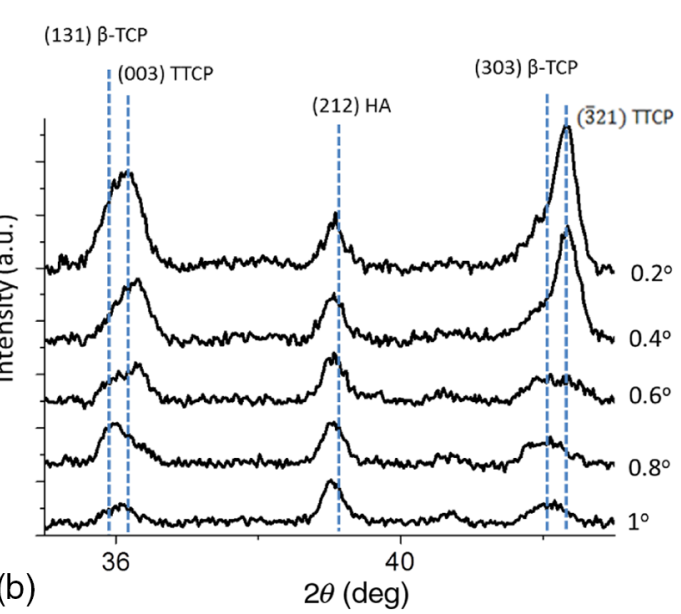

(a)

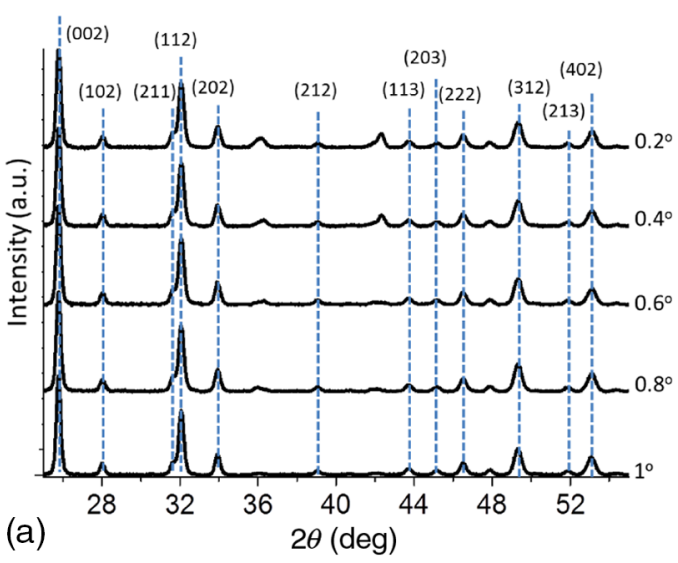

(b)

Fig. 7 (a) Full range x-ray diffraction spectra obtained for different incoming angles of enamel specimen treated with $14 \mathrm{~J} / \mathrm{cm}^{2}$, and (b) the magnified view of the spectra. 
by the different constitution of these tissues. While enamel consists essentially of hydroxyapatite, bone and dentin contain significant amounts of organic compounds and water. ${ }^{49,50}$ Moreover, the cohesion of dentin and bone is ensured by a network of collagen fibrils, reinforced by hydroxyapatite nanocrystals. Collagen presents an ablation threshold much lower than the ablation threshold of hydroxyapatite $\left(0.06 \mathrm{~J} / \mathrm{cm}^{251}\right.$ as compared to $3.3 \mathrm{~J} / \mathrm{cm}^{252}$ ). Therefore, the cohesive strength of bone and dentin can be reduced at lower fluences, facilitating the tissue's removal.

The surface of the specimens treated with a scanning laser beam was covered by ablation debris, which could be removed by ultrasonication, revealing a layer of resolidified material underneath. The number of pulses per surface position in these experiments was 403 , which corresponds to an ablation threshold value of $0.48 \mathrm{~J} / \mathrm{cm}^{2}$, calculated using Eq. (4). Since the fluences used were significantly higher than this threshold, intense ablation was expected, leading to the ejection of a large amount of ablation debris, which redeposited on the surface after the collapse of the ablation plume ${ }^{45}$ forming a continuous layer of material loosely adherent to the surface. Below this layer of ablation debris, a very thin layer of resolidified material exists, which for the specimen treated with $14 \mathrm{~J} / \mathrm{cm}^{2}$ presents a thickness varying within the range of 300 to $900 \mathrm{~nm}$.

Since the ablation debris and the resolidified material were formed by rapid cooling of calcium phosphate from the liquid phase, products such as ACP, tricalcium phosphate, and tetracalcium phosphate can be expected. ${ }^{53}$ The present results show that the ablation debris and the resolidified material contain ACP, formed by rapid solidification of hydroxyapatite. Traces of $\beta$-TCP were detected by $\mathrm{x}$-ray diffraction in the specimen treated with $14 \mathrm{~J} / \mathrm{cm}^{2}$, but this identification needs to be confirmed. No other decomposition products of hydroxyapatite were detected in laser-treated surfaces.

\section{Conclusions}

Enamel can be effectively ablated using a femtosecond laser with 560 -fs pulse duration and $1030-\mathrm{nm}$ wavelength. The single pulse of the ablation threshold is $3.3 \mathrm{~J} / \mathrm{cm}^{2}$. The ablation threshold of enamel decreases with increasing number of laser pulse indicating an incubation effect characterized by an incubation coefficient of 0.68 . The ablation surfaces are smooth and present no evidence of carbonization or cracking. They are covered by a layer of resolidified material, whose thickness increases with increasing fluence. The morphology of the resolidified material indicates that hydroxyapatite gaseous decomposition products (probably $\mathrm{CO}_{2}$ and $\mathrm{H}_{2} \mathrm{O}$ ) were released within the liquid. In the specimens treated with a scanning laser beam, the resolidified layer is covered by a layer of ablation debris loosely attached to the surfaces, which can be partially removed by ultrasonication. The thickness of the resolidified material layer varied between 300 and $900 \mathrm{~nm}$ in the specimen treated with $14 \mathrm{~J} / \mathrm{cm}^{2}$. Below this thin altered layer, the structure and composition of enamel is preserved. ACP was detected in the ablation debris and in the resolidified material. On the other hand, a new phase, probably $\beta$-tricalcium phosphate $\mathrm{Ca}_{3}\left(\mathrm{PO}_{4}\right)_{2}$, was observed at the surface of the specimen treated with $14 \mathrm{~J} / \mathrm{cm}^{2}$. The present results show that the ablation of enamel involves the melting of hydroxyapatite, which is the predominant phase in enamel, but the thickness of the altered layer is very small and thermal damage of the remaining material is negligible.

\section{Acknowledgments}

Q. T. Le gratefully acknowledges a $\mathrm{PhD}$ grant of the Erasmus Mundus Program International Doctoral School of Functional Materials (IDS FunMat) Project No. 2013-14.

\section{References}

1. V. B. Krapchev, C. D. Rabii, and J. A. Harrington, "Novel $\mathrm{CO}_{2}$ laser system for hard tissue ablation," Proc. SPIE 2128, 341 (1994).

2. D. Fried et al., "Multiple pulse irradiation of dental hard tissues at $\mathrm{CO}_{2}$ laser wavelengths," Proc. SPIE 2394, 41 (1995).

3. M. Staninec et al., "Pulpal effects of enamel ablation with a microsecond pulsed lambda $=9.3$-microm $\mathrm{CO}_{2}$ laser," Lasers Surg. Med. 41, 256-263 (2009).

4. I. W. Jeffrey et al., "Dentinal temperature transients caused by exposure to $\mathrm{CO}_{2}$ laser irradiation and possible pulpal damage," J. Dent. 18, 31-36 (1990).

5. D. M. Harris et al., "Selective ablation of surface enamel caries with a pulsed Nd:YAG dental laser," Lasers Surg. Med. 30, 342-350 (2002).

6. V. Armengol, A. Jean, and D. Marion, "Temperature rise during Er : YAG and Nd : YAP laser ablation of dentin," J. Endod. 26, 138-141 (2000).

7. Y. Launay et al., "Thermal effects of lasers on dental tissues," Lasers Surg. Med. 7, 473-477 (1987).

8. A. McDonald et al., "The effect of Nd : YAG radiation at nanosecond pulse duration on dentine crater depth," Biomaterials 23, 51-58 (2002).

9. S. Kuroda and B. O. Fowler, "Compositional, structural, and phase changes in in vitro laser-irradiated human tooth enamel," Calcif. Tissue Int. 36, 361-369 (1984).

10. J. M. White, "Ablation rate, caries removal, and restoration using Nd: YAG and Er:YAG lasers and air abrasion," Proc. SPIE 3248, 98 (1998).

11. S. R. Visuri, J. T. Walsh, and H. A. Wigdor, "Erbium laser ablation of dental hard tissue: effect of water cooling," Lasers Surg. Med. 18, 294-300 (1996)

12. L. Ceballo et al., "Bonding to Er-YAG-laser-treated dentin," J. Dent. Res. 81, 119-122 (2002).

13. L. Bachmann et al., "Changes in chemical composition and collagen structure of dentine tissue after erbium laser irradiation," Spectrochim. Acta A Mol. Biomol. Spectrosc. 61, 2634-2639 (2005).

14. Y. Nishimoto et al., "Effect of pulse duration of Er : YAG laser on dentin ablation," Dent. Mater. J. 27, 433-439 (2008).

15. D. Fried, J. Ragadio, and A. Champion, "Residual heat deposition in dental enamel during IR laser ablation at 2.79, 2.94, 9.6, and 10.6 microm," Lasers Surg. Med. 29, 221-229 (2001).

16. L. Bachmann et al., "Crystalline structure of human enamel irradiated with Er, Cr:YSGG laser," Laser Phys. Lett. 6, 159-162 (2009).

17. M. Mir et al., "Influence of water-layer thickness on Er:YAG laser ablation of enamel of bovine anterior teeth," Lasers Med. Sci. 23, 451-457 (2008).

18. L. E. Rodriguez-Vilchis et al., "Morphological and structural changes on human dental enamel after Er:YAG laser irradiation: AFM, SEM, and EDS evaluation," Photomed. Laser Surg. 29, 493-500 (2011).

19. B. S. Lee, Y. L. Hung, and W. H. Lan, "Compositional and morphological changes of human dentin after Er : YAG laser irradiation," Int. Congr. Ser. 1248, 143-152 (2003).

20. S. Eugénio et al., "KrF laser treatment of human dentin," Proc. SPIE 7131, 71311M (2009)

21. K. Hashiguchi and K. Hashimoto, "Effects of $\mathrm{KrF}$ excimer laser irradiation on human dental enamel," Okajimas Folia Anat. Jpn. 76, 321-333 (2000).

22. S. Eugenio et al., "Characterisation of dentin surfaces processed with KrF excimer laser radiation," Biomaterials 26, 6780-6787 (2005).

23. M. Sivakumar et al., "Influence of tubule orientation on cone-shaped texture development in laser-ablated dentin," Lasers Med. Sci. 21, 160-164 (2006).

24. M. Sivakumar et al., "KrF excimer laser ablation of human enamel," Mater. Sci. Forum 587-588, 42-46 (2008).

25. J. Neev et al., "Ultrashort pulse lasers for hard tissue ablation," IEEE J. Sel. Top. Quantum Electron. 2, 790-800 (1996).

26. S. Alves, V. Oliveira, and R. Vilar, "Femtosecond laser ablation of dentin," J. Phys. D. Appl. Phys. 45, 245401 (2012). 
27. A. V. Rode et al., "Precision ablation of dental enamel using a subpicosecond pulsed laser," Aust. Dent. J. 48, 233-239 (2003).

28. L. T. Cangueiro et al., "Femtosecond laser ablation of bovine cortical bone," J. Biomed. Opt. 17, 125005 (2012).

29. A. V. Rode et al., "Subpicosecond laser ablation of dental enamel," J. Appl. Phys. 92, 2153-2158 (2002).

30. G. J. Pearson and A. V. Mcdonald, "Invited review use of infrared and ultra-violet lasers in the removal of dental hard-tissue," Lasers Med. Sci. 9, 227-237 (1994).

31. L. F. Ji et al., "Ti:sapphire femtosecond laser ablation of dental enamel, dentine, and cementum," Lasers Med. Sci. 27, 197-204 (2012).

32. J. M. Liu, "Simple technique for measurements of pulsed Gaussianbeam spot sizes," Opt. Lett. 7, 196-198 (1982).

33. L. M. Machado et al., "D-Scan measurement of ablation threshold incubation effects for ultrashort laser pulses," Opt. Express 20, 4114-4123 (2012).

34. Y. Jee, M. F. Becker, and R. M. Walser, "Laser-induced damage on single-crystal metal surfaces," J. Opt. Soc. Am. B 5, 648-659 (1988).

35. D. M. Zezell et al., "Compositional and crystallographic changes on enamel when irradiated by Nd:YAG or Er, Cr:YSGG lasers and its resistance to demineralization when associated with fluoride," Proc. SPIE 7549, $75490 \mathrm{G}$ (2010).

36. C. J. Liao et al., "Thermal decomposition and reconstitution of hydroxyapatite in air atmosphere," Biomaterials 20, 1807-1813 (1999).

37. M. Weinlaender et al., "Raman microprobe investigation of the calcium phosphate phases of three commercially available plasma-flamesprayed hydroxyapatite-coated dental implants," J. Mater. Sci. Mater. Med. 3, 397-401 (1992).

38. J. P. Simmer and A. G. Fincham, "Molecular mechanisms of dental enamel formation," Crit. Rev. Oral Biol. Med. 6, 84-108 (1995).

39. S. Miro et al., "Raman spectroscopy study of damage induced in fluorapatite by swift heavy ion irradiations," J. Raman Spectrosc. 42, 2036-2041 (2011).

40. S. Saber-Samandari et al., "Micro-Raman spectroscopy shows how the coating process affects the characteristics of hydroxylapatite," Acta Biomater. 9, 9538-9546 (2013).

41. C. Combes and C. Rey, "Amorphous calcium phosphates: synthesis, properties and uses in biomaterials," Acta Biomater. 6, 3362-3378 (2010).
42. J. Kruger, W. Kautek, and H. Newesely, "Femtosecond-pulse laser ablation of dental hydroxyapatite and single-crystalline fluoroapatite," Appl. Phys. A-Mater. Sci. Process. 69, S403-S407 (1999).

43. R. Stoian et al., "Coulomb explosion in ultrashort pulsed laser ablation of Al2O3," Phys. Rev. B 62, 13167-13173 (2000).

44. P. Balling and J. Schou, "Femtosecond-laser ablation dynamics of dielectrics: basics and applications for thin films," Reports Prog. Phys. 76, 36502 (2013).

45. D. S. Ivanov and L. V. Zhigilei, "Combined atomistic-continuum modeling of short-pulse laser melting and disintegration of metal films," Phys. Rev. B 68, 64114 (2003).

46. L. V. Zhigilei, Z. Lin, and D. S. Ivanov, "Atomistic modeling of short pulse laser ablation of metals: connections between melting, spallation, and phase explosion"," J. Phys. Chem. C 113, 11892-11906 (2009).

47. I. Mayer et al., "Thermal decomposition of developing enamel," Calcif. Tissue Int. 46, 254-257 (1990).

48. D. W. Holcomb and R. A. Young, "Thermal decomposition of human tooth enamel," Calcif. Tissue Int. 31, 189-201 (1980).

49. A. L. Boskey, "Bone composition: relationship to bone fragility and antiosteoporotic drug effects," Bonekey Rep. 2, 1-11 (2013).

50. I. M. Low, N. Duraman, and U. Mahmood, "Mapping the structure, composition and mechanical properties of human teeth," Mater. Sci. Eng. C 28, 243-247 (2008).

51. A. A. Oraevsky et al., "Plasma mediated ablation of biological tissues with nanosecond-to-femtosecond laser pulses: relative role of linear and nonlinear absorption," IEEE J. Select. Topics Quantum Electron. 2, 801-809 (1996).

52. K. Ozono and M. Obara, "Tailored ablation processing of advanced biomedical hydroxyapatite by femtosecond laser pulses," Appl. Phys. A 77, 303-306 (2003).

53. R. B. Heimann and R. Wirth, "Formation and transformation of amorphous calcium phosphates on titanium alloy surfaces during atmospheric plasma spraying and their subsequent in vitro performance," Biomaterials 27, 823-831 (2006).

Biographies for the authors are not available. 